\title{
Klotho promoter methylation status and its prognostic value in ovarian cancer
}

\author{
MARYAM H. AL-ZAHRANI ${ }^{1 *}$, FATIMAH M. YAHYA $^{1 *}$, MOURAD ASSIDI $^{2,3}$, \\ ASHRAF DALLOL ${ }^{2,3}$ and ABDELBASET BUHMEIDA ${ }^{2}$ \\ ${ }^{1}$ Biochemistry Department, Faculty of Science, ${ }^{2}$ Center of Excellence in Genomic Medicine Research, \\ ${ }^{3}$ Medical Technology Department, Faculty of Applied Medical Sciences, \\ King Abdulaziz University, Jeddah 21589, Saudi Arabia
}

Received February 14, 2021; Accepted May 21, 2021

DOI: $10.3892 / \mathrm{mco} .2021 .2343$

\begin{abstract}
Among all gynecological cancers, ovarian cancer (OC) is one of the deadliest types of cancer worldwide. Epigenetic silencing of some genes has been reported to be associated with OC. In this context, Klotho (KL) gene methylation is a promising biomarker for OC. The present study aimed to investigate the methylation profiles of $\mathrm{KL}$ and assess its prognostic value. A total of 63 formalin-fixed paraffin-embedded tissue samples from patients with primary OC were collected and analyzed in the present study. The methylation profiles of KL were assessed by performing DNA bisulfate treatment followed by DNA promoter methylation analysis using the MethyLight assay. The results revealed KL promoter hypermethylation in $62 \%$ of the OC cohort. Additionally, significant associations were observed between KL methylation profiles and tumor subtype $(\mathrm{P}<0.0001)$ and tumor site $(\mathrm{P}=0.039)$. Furthermore, Kaplan-Meier analysis revealed that a worse disease-specific survival was significantly associated with hypermethylated $\mathrm{KL}(\mathrm{P}=0.03$, log-rank; hazard ration, 0.58 ; 95\% confidence interval (CI), 0.26-0.90). Cox regression multivariate analysis indicated that KL promoter methylation was an independent OC prognostic indicator $(\mathrm{P}=0.029)$. The current study suggested that KL may be a novel biomarker to predict prognosis in patients with $\mathrm{OC}$, since patients with higher KL promoter methylation were more likely to have a poor prognosis and would therefore require frequent follow-up and integrative personalized therapeutic approaches.
\end{abstract}

Correspondence to: Professor Abdelbaset Buhmeida, Center of Excellence in Genomic Medicine Research, King Abdulaziz University, P.O. Box 80216, Jeddah 21589, Saudi Arabia

E-mail: abuhme@utu.fi

*Contributed equally

Key words: ovarian cancer, Klotho, methylation, prognosis, biomarker, survival

\section{Introduction}

Among the different gynecologic cancers, ovarian cancer (OC) is the leading cause of death (1). In comparison to the breast cancer, OC mortality rate is approximately three times higher despite its lower incidence rate (2). This is attributed to its asymptomatic initial stages and late diagnosis at advanced stages $(3,4)$. The incidence of OC in Saudi Arabia has increased 4-fold between 1990 and 2016 (5). It affects more than 3\% of Saudi women in their lifetime $(6,7)$. There are different types of ovarian malignancy; however, the epithelial ovarian cancer (EOC) is the most common malignancy (90\%) (4,8-10). This EOC is a heterogeneous disease with several histologic subtypes that exhibited distinct cytogenetic features, molecular signatures and onco-pathological signaling pathways. It is a highly invasive with five distinct histologic subtypes: High grade serous which accounts for $70 \%$ of EOC, endometrioid constitutes $10 \%$, clear cell $(10 \%)$, low grade serous $5 \%$ and mucinous $3 \%$ of EOC (5).

With huge milestones being achieved in the genomic analysis, some inherited mutations of specific genes, such as BRCA1 and BRCA2, have been shown to increase OC risk. In fact, genomic events such as amplification (HER2/KRAS) and mutations (KRAS, PIK, RAD, BRAD) have been reported in OC (11-13). In contrast, oral contraceptive pills, plurality, hysterectomy, and breastfeeding have lower OC incidence $(14,15)$.

Studies have shown that the 5-year survival rate of patients with OC depends mainly on the disease stage at diagnosis. In general, patients diagnosed at an early stage (I/II) have higher 5 year survivals than those at late stage (III/IV). Furthermore, a noticeable increase in OC post-relapse survival was reported over the years during the last decades (16). With the advent of high-throughput sequencing technologies, the overall aim of precision medicine for patients with $\mathrm{OC}$ is to predict, prevent, and treat the disease while improving the clinical outcomes and reducing the adverse effects associated with invasive therapeutic approaches (17). Therefore, there is a need for devising new effective strategies based on genomic and epigenomic signatures to identify relevant molecular biomarkers and establish personalized therapeutics $(18,19)$. 
Genomic instability is one of the main causes of cancer onset attributed to the interactions among a patient's susceptible genome, environment, and lifestyle; hence, some DNA alterations occur at the epigenomic level. Epigenetic alterations encompass histone acetylation, noncoding RNAs, and $\mathrm{CpG}$ island methylation, are among the most frequent epigenetic alterations observed in OC (20-22). Currently, there is an increasing interest in examining the specific patterns of hypermethylation of $\mathrm{CpG}$ islands in OC. Both targeted and whole-genome analyses for DNA methylation patterns have shown potential in identifying methylation biomarkers and their prognostic value in OC (23-26). Moreover, epigenetic silencing of some genes, including hMLH1, RASSF1A, and FANCF due to methylation is associated with ovarian tumor drug resistance (27). Consequently, some key epigenetic events are likely to provide cancer cells an advantage to proliferate and invade, thereby promoting metastasis $(28,29)$. Thus, further studies are required to analyze the methylation profiles of several genes to gain deeper insights into the epigenetic alterations in OC, especially in the Arabian Peninsula, where only a few studies have been reported $(25,26,30-32)$. In this context, klotho (KL) gene is an interesting candidate aging marker gene that could be associated with aging-related diseases e.g. cancer. In fact, KL gene was reported with an aberrantly methylated promoter reported in several malignancies (33). Initially discovered as an anti-aging factor (34), KL is located in chromosome $13 \mathrm{q} 12$. It is $50 \mathrm{~kb}$ in length and is composed of five exons and four introns (35). It is highly expressed in the kidneys and brain but can also be expressed in the placenta, breast, ovaries, uterus, and fallopian tube tissues (36). In the female reproductive system, particularly in the ovary, the level of abnormal expression of KL in granulosa cells is tightly linked to the severity of ovary-related diseases (37). KL expression levels decrease in the brain with advancing age in mammals. This decrease could be due to hypermethylation of the promoter region of $\operatorname{KL}(38,39)$. Furthermore, aberrant methylation in the promoter region of KL decreases gene transcription (40). Recently, KL has been reported as a potential tumor suppressor gene that inhibits the IGF-1 pathway and is a novel target gene for epigenetic silencing in cancers of breast (41), cervix (34), stomach (42), bladder, and ovaries (43). However, low expression levels of KL are associated with weakness in skeletal muscle, difficulty in executing daily activities, and increased mortality in the elderly. Deficiency in IGF-1 expression level is a reported feature of aging, and is likely affected by methylated KL (40). The 1439-bp long promoter region of KL lacks a TATA box and instead, contains four potential Sp1 binding sites $(35,44)$. Moreover, KL expression can be restored using demethylation reagents (35). So far, only few studies have assessed the methylation profiles of $\mathrm{KL}$ in $\mathrm{OC}$ and showed potential prognosis value. In that, higher KL promoter methylation profiles were associated with cancer progression $(45,46)$. Therefore, additional studies are required to further understand the prognosis value of KL methylation status in OC, particularly, in the Arabian Peninsula population. In this study, we analyzed KL gene promoter methylation profiles in OC and assessed its prognostic value.

\section{Patients and methods}

Patients and samples. This retrospective study comprised 63 formalin-fixed paraffin-embedded (FFPE) tissues that were surgically resected after obtaining written consent from female patients who were diagnosed with OC and were selected based on the availability of both tissues and patient clinicopathological follow-up data. The FFPE samples were collected between 1995 and 2014 at the Departments of Pathology \& Gynecology, King Abdulaziz University Hospital (KAUH).

The cohort of patients was selected from a total of 100 accessible samples based on the availability of patients' annotated clinicopathological follow-up data, and the quality/quantity of extracted DNA. Patients' cohort was classified based on histopathological features using the tumor node metastasis (TNM) classification system.

The main clinicopathological features (such as age, menopausal status, stage, grade, and lymph node status) and follow-up and survival data are summarized in Table I. The samples and data were retrieved from the patients after obtaining all the relevant ethical approvals according to the guidelines of the Ethical Committee of King Abdulaziz University Hospital (Ref. number: KAU-189-14).

DNA extraction. Before DNA extraction, $10 \mu \mathrm{m}$ thin FFPE slices were deparaffinized using xylol (Sigma-Aldrich) and then washed with ethanol 99-100\% (Sigma-Aldrich). DNA extraction was performed using the QIAamp DNA FFPE Tissue Kit (Qiagen) according to the manufacturer's protocol. The quality and concentration of all DNA samples were assessed using NanoDrop 2000 Spectrophotometer (Thermo Fisher Scientific, Inc.).

KLmethylation analysis. Bisulfite treatment of the KL promoter region was performed using the Qiagen EpiTect ${ }^{\circledR}$ Bisulfite Conversion kit as detailed in the manufacturer's handbook and reported by Dallol et al (47). Briefly, $0.5 \mu \mathrm{g}$ extracted DNA was incubated with $\mathrm{NaH}_{2} \mathrm{SO}_{4}(3.12 \mathrm{M})$. Only the unmethylated cytosine $(\mathrm{C})$ residues were converted into uracil (U) residues. The MethyLight assay for the candidate gene was performed using the EpiTect ${ }^{\circledR}$ MethyLight PCR Kit (Qiagen, Inc.) and EpiTect ${ }^{\circledR}$ Control DNA Set (Qiagen, Inc.) according to the protocol guidelines, fluorescent dual-labeled TaqMan Probe 6FAM-CGGTTGGGTTAATCGCGTTTT-BHQ1, and specifically designed primers: Forward primer 5'-AGCGTTTGT AGGACGTTTAC-3', and reverse primer 5'-TAACGAAAA CAAAACTCCGC-3') (48); qPCR was performed using the StepOnePlus $^{\mathrm{TM}}$ real-time PCR system (Applied Biosystems).

Statistical analysis. Statistical analyses were performed using SPSS $^{\circledR}$ version 19 (IBM Corp.). Differentially hypo/hypermethylated genes' profiles were assessed compared to the EpiTect ${ }^{\circledR}$ Control DNA Set using a t-test. The association between KL methylation profile and clinicopathological features of patients was analyzed using Fisher-Freeman-Halton Exact test. Furthermore, disease-specific survival (DSS) and disease-free survival (DFS) based on KL methylation profiles were calculated by univariate Kaplan-Meier analysis along with hazard ration calculation at $95 \% \mathrm{CI}$, and equality of the 
Table I. Association between KL methylation profiles and clinicopathological features of patients with ovarian cancer.

\begin{tabular}{|c|c|c|c|c|}
\hline \multirow[b]{2}{*}{ Clinicopathological features } & \multirow[b]{2}{*}{$\mathrm{N}(\%)$} & \multicolumn{2}{|c|}{ KL methylation profile } & \multirow[b]{2}{*}{ P-value } \\
\hline & & 0 unmethylated, n (\%) & 1 hypermethylated, n (\%) & \\
\hline \multicolumn{5}{|l|}{ Age, years } \\
\hline$<50$ & $40(63.5)$ & $19(79.2)$ & $21(55.3)$ & 0.064 \\
\hline$>50$ & $22(34.9)$ & $5(20.8)$ & $17(44.7)$ & \\
\hline NA & 1 (1.6) & & & \\
\hline \multicolumn{5}{|l|}{ Diagnosis method } \\
\hline Surgery & $58(92.1)$ & $22(91.7)$ & $36(94.7)$ & 0.637 \\
\hline Biopsy & $4 \quad(6.3)$ & $2(8.3)$ & $2(5.3)$ & \\
\hline NA & 1 (1.6) & & & \\
\hline \multicolumn{5}{|l|}{ Tumor site } \\
\hline Right & $11(17.5)$ & $4(16.7)$ & $7(18.4)$ & $0.039^{\mathrm{b}}$ \\
\hline Left & $11(17.5)$ & $8(33.3)$ & 3 (7.9) & \\
\hline Bilateral & $40(63.5)$ & $12(50.0)$ & $28(73.7)$ & \\
\hline NA & 1 (1.6) & & & \\
\hline \multicolumn{5}{|l|}{ Tumor size, $\mathrm{cm}$} \\
\hline $1-5$ & $18(28.6)$ & $9(39.1)$ & $9(23.7)$ & 0.370 \\
\hline $6-10$ & $16(25.4)$ & $6(26.1)$ & $10(26.3)$ & \\
\hline$>10$ & $27(42.9)$ & $8(34.8)$ & $19(50.0)$ & \\
\hline NA & $2(3.2)$ & & & \\
\hline \multicolumn{5}{|l|}{ Tumor subtype } \\
\hline Serous & $30(47.6)$ & $12(50.0)$ & $18(47.4)$ & $<0.001^{\mathrm{b}}$ \\
\hline Mucinous & $17(27.0)$ & 1 (4.2) & $16(42.1)$ & \\
\hline Other & $14(22.2)$ & $11(45.8)$ & 3 (7.9) & \\
\hline NA & $2(3.2)$ & $0 \quad(0.0)$ & 1 (2.6) & \\
\hline \multicolumn{5}{|l|}{ Tumor grade } \\
\hline Undetermined grade (GX) & $10(15.9)$ & $5(23.8)$ & $5(16.1)$ & 0.825 \\
\hline Low malignant potential (GB) & $5 \quad(7.9)$ & $2(9.5)$ & $3(9.7)$ & \\
\hline Well-differentiated (G1) & $3(4.8)$ & $2(9.5)$ & $1 \quad(3.2)$ & \\
\hline Moderately differentiated (G2) & $15(23.8)$ & $5(23.8)$ & $10(32.3)$ & \\
\hline Undifferentiated (G3 to G4) & $19(30.2)$ & $7(33.3)$ & $12(38.7)$ & \\
\hline NA & $11(17.5)$ & & & \\
\hline \multicolumn{5}{|l|}{ Lymph node status } \\
\hline Positive & $11(17.5)$ & $2(22.2)$ & $9(56.3)$ & 0.208 \\
\hline Negative & $14(22.2)$ & $7(77.8)$ & $7(43.8)$ & \\
\hline NA & $38(60.3)$ & & & \\
\hline \multicolumn{5}{|l|}{ Body mass index } \\
\hline$<23$ & $2(3.2)$ & $0 \quad(0.0)$ & $2(9.5)$ & 0.118 \\
\hline $23-26$ & $12(19.0)$ & $8(47.1)$ & $4(19.0)$ & \\
\hline$>26$ & $24(38.1)$ & $9(52.9)$ & $15(71.4)$ & \\
\hline NA & $25(39.7)$ & & & \\
\hline \multicolumn{5}{|l|}{ Parity } \\
\hline Parous & $29(46.0)$ & $10(62.5)$ & $19(73.1)$ & 0.510 \\
\hline Nulliparous & 13 (20.6) & $6(37.5)$ & 7 (26.9) & \\
\hline NA & $21(33.3)$ & & & \\
\hline \multicolumn{5}{|l|}{ Menopausal status } \\
\hline Premenopausal & $41(65.1)$ & $19(79.2)$ & $22(57.9)$ & 0.104 \\
\hline Postmenopausal & $21(33.3)$ & $5(20.8)$ & $16(42.1)$ & \\
\hline NA & 1 (1.6) & & & \\
\hline
\end{tabular}


Table I. Continued.

\begin{tabular}{|c|c|c|c|c|}
\hline \multirow[b]{2}{*}{ Clinicopathological features } & \multirow[b]{2}{*}{$\mathrm{N}(\%)$} & \multicolumn{2}{|c|}{ KL methylation profile } & \multirow[b]{2}{*}{ P-value } \\
\hline & & 0 unmethylated, n (\%) & 1 hypermethylated, n (\%) & \\
\hline \multicolumn{5}{|l|}{ Tumor stage } \\
\hline Stage I & $16(25.4)$ & $8(38.1)$ & $8(22.9)$ & 0.660 \\
\hline Stage II & $4 \quad(6.3)$ & $1 \quad(4.8)$ & $3(8.6)$ & \\
\hline Stage III & $31(49.2)$ & $10(47.6)$ & $21(60.0)$ & \\
\hline Stage IV & $5 \quad(7.9)$ & $2(9.5)$ & $3(8.6)$ & \\
\hline NA & $7(11.1)$ & & & \\
\hline \multicolumn{5}{|l|}{ Endpoint status } \\
\hline Living & $24(38.1)$ & $11(73.3)$ & $13(41.9)$ & 0.063 \\
\hline Deceased & $22(34.9)$ & $4(26.7)$ & $18(58.1)$ & \\
\hline NA & $17(27.0)$ & & & \\
\hline \multicolumn{5}{|l|}{ Recurrence status } \\
\hline No & $20(31.7)$ & $7(41.2)$ & $13(48.1)$ & 0.760 \\
\hline Yes & $24(83.1)$ & $10(58.8)$ & $14(51.9)$ & \\
\hline NA & $19(30.2)$ & & & \\
\hline
\end{tabular}

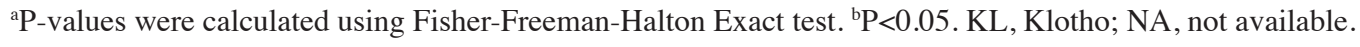

survival functions were determined by log-rank (Mantel-Cox) test. Additionally, Cox-regression multivariate analysis was performed to assess possible independent prognostic impact. Results with $\mathrm{P}<0.05$ were considered statistically significant.

\section{Results}

Patient cohort overview. As shown in Table I, most of our patients diagnosed with OC $(64.5 \%)$ were $<50$ years of age. Additionally, most patients $(66.1 \%)$ were premenopausal women. Furthermore, a higher number of patients in our cohort were parous women. Notably, a high proportion of patients were diagnosed with OC at advanced stages (64.3\%) and a high grade $(36.5 \%)$, where the size of the eradicated tumors was $>10 \mathrm{~cm}^{3}$. Moreover, a high percentage of our cohort was diagnosed with the serous histological subtype; incidentally, among these, many women were obese with BMI >26 (Table I).

KL methylation profiles and clinicopathological features. Our results revealed highly frequent methylation of KL among OC patients $(n=39,61.9 \%)$. Further, correlation analysis between KL methylation profile and clinicopathol ogical features of our cohort using Fisher-Freeman-Halton Exact test revealed significant associations between KL methylation pattern and tumor subtype $(\mathrm{P}<0.0001)$, tumor site $(\mathrm{P}=0.036)$, and borderline significance for endpoint status $(\mathrm{P}=0.063)$, and age $(\mathrm{P}=0.064)$ (Table I). In fact, KL promoter methylation was observed in the majority (77\%) of old OC patients (>50 years). Particularly, this KL hypermethylation was more prevalent (70\%) in bilateral OC patients. However, no significant associations with grade, BMI, age, and stage $(\mathrm{P}>0.05)$ were observed.

KL methylation profiles and survival outcomes. Kaplan-Meier analysis of DSS revealed a significant difference between patients with OC showing high KL methylation, who lived for a significantly shorter duration (shorter DSS) than those with non-methylated KL using the cut-off [unmethylated ( 0 ; 15 cases) vs. hypermethylated (1;31 cases)] as a determinant of DSS in univariate (Kaplan-Meier) analysis (Fig. 1; $\mathrm{P}=0.03$, log-rank). For example, in a 1-year follow-up period, all patients with non-methylated KL (100\%) were alive compared to only $75 \%$ of those with methylated KL. Similarly, in the 5 -year follow-up period, $40 \%$ of the patients with OC who had methylated KL, had died, whereas, $85 \%$ of those with non-methylated KL were still alive. Our DSS results showed a hazard ratio $=0.58$; $(95 \% \mathrm{CI}, 0.26-0.90)$; This means that at any follow time, the OC patients with methylated KL group has 0.58 times as likely more than the control (unmethylated) KL group. Which means that patients with unmethylated KL has 0.42 times to die less than the KL methylated group. Therefore, $\mathrm{OC}$ with methylated KL is more aggressive and a predictive indicator of poor prognosis in OC patients. Furthermore, a slight trend of reduced recurrence was observed in OC patients with non-methylated KL. In fact, DFS analysis using the cut-off [unmethylated $(0 ; 17$ cases) vs. hypermethylated $(1 ; 27$ cases)] as a determinant of DFS in univariate (Kaplan-Meier) analysis showed no significant differences between patients with non-methylated KL, and those with hypermethylated KL and living with no recurrence [Fig. 2; $\mathrm{P}=0.3$, log-rank, hazard ratio=1.17; (95\% CI, 0.78-1.56)].

Multivariate analysis. A multivariable analysis was carried out to determine if the survival association is independent of other factors. In fact, multivariate Cox's regression analysis suggests that KL methylation is an independent poor survival marker $(\mathrm{P}=0.029)$ in relation to patient's age, tumor grade and most importantly independent of the histological subtype of the tumor (Table II). 
Table II. Cox regression analysis of the prognostic values of KL methylation, age at diagnosis, grade and histological subtypes.

\begin{tabular}{lcccr}
\hline Feature & P-value & Standard error value & Relative risk & $95 \%$ CI \\
\hline KL methylation (low vs. high methylation) & 0.029 & 0.716 & 4.758 & $1.169-19.366$ \\
Age at diagnosis (<50 vs. >50 years) & 0.843 & 0.540 & 1.113 & $0.386-3.205$ \\
Histological subtypes (serous vs. mucinous) & 0.470 & 0.296 & 1.238 & $0.693-2.213$ \\
Tumor grade (low vs. high grade) & 0.140 & 0.186 & 1.317 & $0.914-1.898$ \\
\hline
\end{tabular}

KL, Klotho; CI, confidence interval.

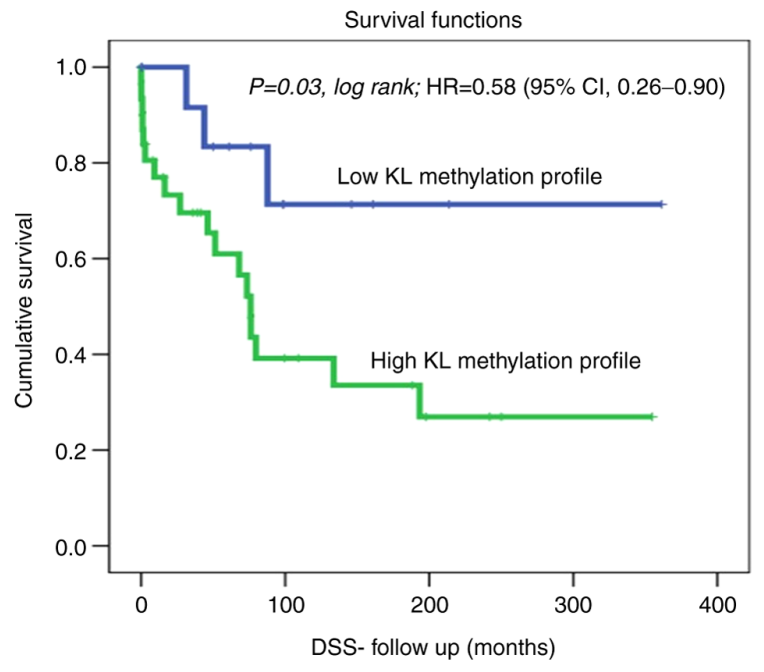

Figure 1. KL methylation profile status in the ovarian cancer cohort using the cut-off [unmethylated $(0 ; 15$ cases) vs. hypermethylated $(1 ; 31$ cases $)$ ] as a determinant of DSS in univariate (Kaplan-Meier) analysis. DSS, disease-specific survival; KL, Klotho; HR, hazard ratio.

\section{Discussion}

$\mathrm{OC}$ is one of the deadliest gynecological cancers mainly because of delayed diagnosis and asymptomatic early-stage progression. Moreover, the deleterious effects of OC can be attributed to the anatomical position of the ovaries within the deep pelvic cavity which may hide the disease expansion and delay the diagnosis (49-51). Scientists are seeking to improve the detection, prognosis, management, and treatment approaches toward precision oncology to alleviate the burden of this disease on patients, their families, and the healthcare system (52). With the development of high-throughput sequencing technologies, it is possible to assess changes in the genetic and epigenetic profiles of the target genes/genomes and identify potential molecular biomarkers for OC (21,53-56). $\mathrm{KL}$ is located at chromosome $13 \mathrm{q} 12$, with a length of $50 \mathrm{~kb}$ including a promoter region of $1,439 \mathrm{bp}$. It is a known tumor suppressor gene that has been reported to undergo aberrant promoter methylation in many tumors (35). The level of abnormal expression of KL in granulosa cells of the ovary is tightly linked with the acuteness of ovary-related diseases (37). Thus, in this study, the methylation pattern of $\mathrm{KL}$ were determined to evaluate its potential as a promising molecular biomarker in $\mathrm{OC}$ and assess its prognostic value. To the best of our knowledge, this is the first study conducted

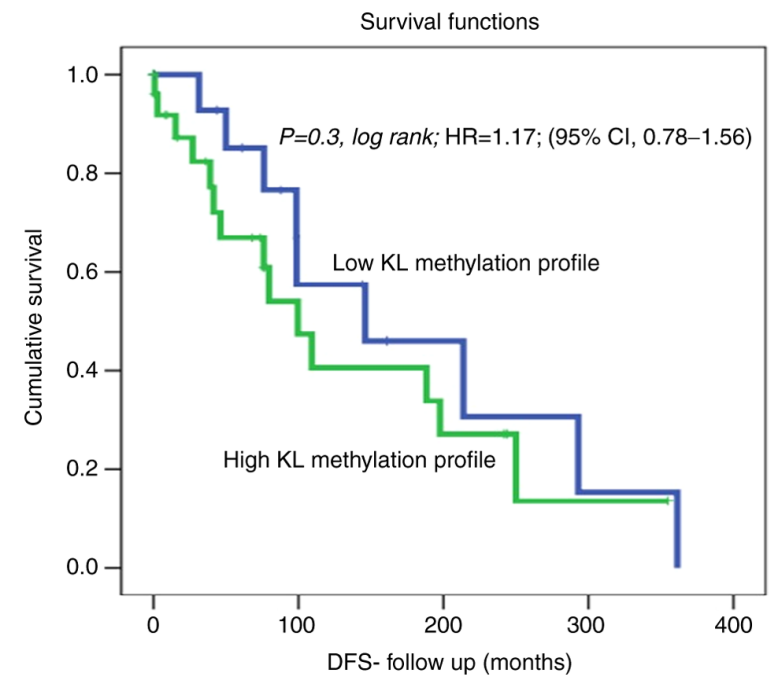

Figure 2. KL methylation profile status in the ovarian cancer cohort using the cut-off [unmethylated $(0 ; 17$ cases) vs. hypermethylated $(1 ; 27$ cases)] as a determinant of DFS in univariate (Kaplan-Meier) analysis. DFS, disease-free survival; KL, Klotho; HR, hazard ratio.

in the Arabian Peninsula to assess this biomarker in patients with OC and particular clinicopathological features. Unlike previous studies, most of our cohort was diagnosed with OC before menopause $(66.1 \%)$ and was younger than 50 years of age $(64.5 \%)$. In contrast, other studies reported OC as an age-related disease, and as mainly postmenopausal (57). Moreover, the incidence increased more noticeably in women over 65 years of age (58), and the median age at diagnosis was between 50 and 79 years $(59,60)$. According to the data released by Cancer Research UK, $53 \%$ of British women diagnosed with OC were 65 years and older. Moreover, according to the American Cancer Society report, $50 \%$ of the diagnosed OC patients were aged 63 years or above (61). These findings highlight an interesting 15-year shift in the onset of $\mathrm{OC}$ in the Arabian Peninsula, and therefore, warrant additional studies to demystify such an early onset. Parity has been reported to reduce the risk of OC in contrast to nulliparous women (62). However, the majority of our patient cohort was parous women (69\%), which may be attributed to the genomic blueprint, environmental factors, and/or lifestyle choices; however, this needs further investigation.

Till date, only Wiley et al have assessed the KL methylation profiles in 215 epithelial OC patients. They found that KL was hypermethylated in $14 \%$ of the OC patients compared with 
the controls and the hypermethylation status was associated with a significant reduction in DFS (46). Strikingly, approximately $62 \%$ of our cohort had hypermethylated KL, which is 4-fold higher than the results reported by Wiley et al (46). Our results showed significant associations between KL methylation profile and tumor subtype $(\mathrm{P}<0.0001)$. Different OC subtypes have been established with distinct molecular signatures $(63,64)$. Our data showed KL promoter hypermethylation mainly in mucinous and serous subtypes. KL methylation showed borderline significance with age $(\mathrm{P}=0.064)$ (Table I). In contrast to the findings of Wiley et al (46), analysis of KL methylation profiles of OC patients did not reveal a significant correlation with DFS in our cohort (Fig. 2); however, a trend of faster recurrence in patients with KL promoter methylation was observed (Fig. 2). Moreover, DSS was significantly associated with KL methylation ( $\mathrm{P}=0.03$; log-rank test; Fig. 1). Our data showed that patients with higher KL promoter methylation were more likely to die earlier than those with non-methylated KL. In fact, in a 1-year follow-up, $75 \%$ of the patients with methylated KL were alive compared to those with non-methylated KL $(100 \%)$. These results are in line with those of other tumors, mainly gastric, hepatocellular, and cervical carcinoma, where KL methylation profiles are associated with poor DFS $(33,34,42)$. However, despite the association between downregulation of KL expression in follicular cells and ovarian diseases (37), the molecular mechanism of KL action and its relationship with aging and other diseases remains poorly understood and requires further investigation.

We acknowledge that our current OC study is a pilot study that gives clues about KL methylation prognosis value, and represents only the cohort of participants with possible inherent and unavoidable bias due to the size of the cohort and the available biological material and associated follow-up data. A large-scale multi-centric validation study is required to confirm these findings.

Our results showed that KL promoter hypermethylation is a poor prognosticator in OC patients. Patients with higher KL promoter methylation are more likely to die earlier, and therefore, more vigilant follow-up and integrative therapeutic approaches are required. Due to the increase in OC related mortality and the absence of early diagnosis or effective therapeutics, KL methylation could be used as an OC prognosticator to achieve better and personalized clinical management of this gynecological malignancy.

\section{Acknowledgements}

Not applicable.

\section{Funding}

The present study was supported by the Deanship of Scientific Research in KAU (grant no. G:205-247-1440).

\section{Availability of data and materials}

The datasets used and/or analyzed during the current study are available from the corresponding author on reasonable request.

\section{Authors' contributions}

MHAZ and AB contributed to study design, statistical analysis and contributed in both drafting and critical revision of the manuscript. FMY and MA contributed to data collection, performing the experiments and manuscript drafting. $\mathrm{AD}$ performed part of the experiments and contributed to data analysis. AB and MA have checked and confirmed the authenticity of the data. All authors critically reviewed and approved the final version of the manuscript.

\section{Ethics approval and consent to participate}

The samples and data were retrieved from the patients after obtaining all the relevant ethical approvals according to the guidelines of the Ethical Committee of King Abdulaziz University Hospital (Jeddah, Saudi Arabia; ref. no. KAU-189-14). Written informed consent was obtained from all patients.

\section{Patient consent for publication}

Not applicable.

\section{Competing interests}

The authors declare that they have no competing interests.

\section{References}

1. Torre LA, Trabert B, DeSantis CE, Miller KD, Samimi G, Runowicz CD, Gaudet MM, Jemal A and Siegel RL: Ovarian Cancer Statistics, 2018. CA Cancer J Clin 68: 284-296, 2018.

2. Yoneda A, Lendorm ME, Couchman JR and Multhaupt HA: Breast and ovarian cancers: A survey and possible roles for the cell surface heparan sulfate proteoglycans. J Histochem Cytochem 60: 9-21, 2012.

3. Lage H and Denkert C: Resistance to chemotherapy in ovarian carcinoma. In: Targeted Therapies in Cancer. Dietel M (ed). Springer Berlin Heidelberg, Berlin, Heidelberg, pp51-60, 2007.

4. Taylor SE and Kirwan JM: Ovarian cancer-current management and future directions. Obstet Gynaecol Reprod Med 19: 130-135, 2009.

5. Althubiti MA and Nour Eldein MM: Trends in the incidence and mortality of cancer in Saudi Arabia. Saudi Med J 39: 1259-1262, 2018.

6. Ferlay J, Colombet M, Soerjomataram I, Mathers C, Parkin DM, Piñeros M, Znaor A, Bray F: Estimating the global cancer incidence and mortality in 2018: GLOBOCAN sources and methods. Int J Cancer 144: 1941-1953, 2019.

7. Almutairi AA, Edali AM, Khan SA, Aldihan WA and Alkhenizan AH: Yield of prostate cancer screening at a community based clinic in Saudi Arabia. Saudi Med J, 40: 681-686, 2019.

8. Karst AM and Drapkin R: Ovarian cancer pathogenesis: A model in evolution. J Oncol 2010: 932371, 2010.

9. Prat J: New insights into ovarian cancer pathology. Ann Oncol 23 (Suppl 10): x111-x117, 2012.

10. Bower M and Waxman J: Ovarian cancer. In: Lecture Notes: Oncology. 3rd edition. Wiley and Sons, London, pp.186-192, 2016.

11. Manna PR, Molehin D and Ahmed AU: Dysregulation of aromatase in breast, endometrial, and ovarian cancers: An overview of therapeutic strategies. In: Molecular and Cellular Changes in the Cancer Cell. Vol. 144. Pruitt K (ed). Elsevier Academic Press Inc., San Diego, CA, pp487-537, 2016.

12. Hesketh PJ, Kris MG, Basch E, Bohlke K, Barbour SY, Clark-Snow RA, Danso MA, Dennis K, Dupuis LL, Dusetzina SB, et al: Antiemetics: American Society of Clinical Oncology clinical practice guideline update. J Clin Oncol 35: 3240-3261, 2017 
13. Norquist BM, Brady MF, Harrell MI, Walsh T, Lee MK, Gulsuner S, Bernards SS, Casadei S, Burger RA, Tewari KS, et al: Mutations in homologous recombination genes and outcomes in ovarian carcinoma patients in GOG 218: An NRG oncology/gynecologic oncology group study. Clin Cancer Res 24: 777-783, 2018

14. Maradeo ME and Cairns P: Translational application of epigenetic alterations: Ovarian cancer as a model. FEBS Lett 585: 2112-2120, 2011

15. Salehi F, Dunfield L, Phillips KP, Krewski D and Vanderhyden BC: Risk factors for ovarian cancer: An overview with emphasis on hormonal factors. J Toxicol Environ Health B Crit Rev 11: 301-321, 2008

16. Irodi A, Rye T, Herbert K, Churchman M, Bartos C, Mackean M, Nussey F, Herrington CS, Gourley C and Hollis RL: Patterns of clinicopathological features and outcome in epithelial ovarian cancer patients: 35 years of prospectively collected data. BJOG 127: 1409-1420, 2020

17. Sapiezynski J, Taratula O, Rodriguez-Rodriguez L and Minko T: Precision targeted therapy of ovarian cancer. J Control Release 243: 250-268, 2016.

18. Currie G and Delles C: Precision medicine and personalized medicine in cardiovascular disease. In: Sex-Specific Analysis of Cardiovascular Function. Vol. 1065. Kerkhof PLM and Miller VM (eds). Springer International Publishing AG, Cham, pp589-605, 2018.

19. Schmid BC and Oehler MK: New perspectives in ovarian cancer treatment. Maturitas 77: 128-136, 2014.

20. Keita M, Wang ZQ, Pelletier JF, Bachvarova M, Plante M, Gregoire J, Renaud MC, Mes-Masson AM, Paquet ÉR and Bachvarov D: Global methylation profiling in serous ovarian cancer is indicative for distinct aberrant DNA methylation signatures associated with tumor aggressiveness and disease progression. Gynecol Oncol 128: 356-363, 2013.

21. Ganzfried BF, Riester M, Haibe-Kains B, Risch T, Tyekucheva S, Jazic I, Wang XV, Ahmadifar M, Birrer MJ, Parmigiani G, et al: curatedOvarianData: Clinically annotated data for the ovarian cancer transcriptome. Database (Oxford) 2013: bat013, 2013.

22. Rajendram R, Rajendram R, Patel VB, Preedy VR: Biomarkers in health and disease: Cancer further knowledge. In: Biomarkers in Cancer. Biomarkers in Disease: Methods, Discoveries and Applications. Preedy V, Patel V (eds). Springer, Dordrecht, pp981-986, 2015.

23. Strathdee G, Appleton K, Illand M, Millan DW, Sargent J, Paul J and Brown R: Primary ovarian carcinomas display multiple methylator phenotypes involving known tumor suppressor genes. Am J Pathol 158: 1121-1127, 2001.

24. Wu JH, Liang XA, Wu YM, Li FS and Dai YM: Identification of DNA methylation of SOX9 in cervical cancer using methylated-CpG island recovery assay. Oncol Rep 29: 125-132, 2013.

25. Lee MP and Dunn BK: Influence of genetic inheritance on global epigenetic states and cancer risk prediction with DNA methylation signature: Challenges in technology and data analysis Nutr Rev 66 (Suppl 1): S69-S72, 2008.

26. Shen SC, Liao CH, Lo YF, Tsai HP, Kuo WL, Yu CC, Chao TC, Chen MF, Chang HK, Lin YC, et al: Favorable outcome of secondary axillary dissection in breast cancer patients with axillary nodal relapse. Ann Surg Oncol 19: 1122-1128, 2012.

27. Balch C, Huang TH, Brown R and Nephew KP: The epigenetics of ovarian cancer drug resistance and resensitization. Am J Obstet Gynecol 191: 1552-1572,2004

28. Esteller M: Epigenetics in cancer. N Engl J Med 358: 1148-1159, 2008

29. Herman JG and Baylin SB: Gene silencing in cancer in association with promoter hypermethylation. N Engl J Med 349: 2042-2054,2003.

30. Ahluwalia A, Yan P, Hurteau JA, Bigsby RM, Jung SH, Huang TH and Nephew KP: DNA methylation and ovarian cancer. I. Analysis of $\mathrm{CpG}$ island hypermethylation in human ovarian cancer using differential methylation hybridization. Gynecol Oncol 82: 261-268, 2001

31. Zeller C, Dai W, Curry E, Siddiq A, Walley A, Masrour N, Kitsou-Mylona I, Anderson G, Ghaem-Maghami S, Brown R and El-Bahrawy M: The DNA methylomes of serous borderline tumors reveal subgroups with malignant- or benign-like profiles. Am J Pathol 182: 668-677, 2013

32. Shi H, Wei SH, Leu YW, Rahmatpanah F, Liu JC, Yan PS, Nephew KP and Huang TH: Triple analysis of the cancer epigenome: An integrated microarray system for assessing gene expression, DNA methylation, and histone acetylation. Cancer Res 63: 2164-2171, 2003 .
33. Xie B, Zhou J, Yuan L, Ren F, Liu DC, Li Q and Shu G: Epigenetic silencing of Klotho expression correlates with poor prognosis of human hepatocellular carcinoma. Hum Pathol 44: 795-801, 2013.

34. Lee J, Jeong DJ, Kim J, Lee S, Park JH, Chang B, Jung SI, Yi L, Han Y, Yang Y, et al: The anti-aging gene Klotho is a novel target for epigenetic silencing in human cervical carcinoma. Mol Cancer 9: 109, 2010.

35. Rubinek T and Wolf I: Klotho tumor suppressor. In: Encyclopedia of Cancer. Schwab M (ed). Springer, Berlin, Heidelberg, pp1-5, 2016.

36. Fan CF, Tan $\mathrm{C}$ and Wang SQ: $\alpha$-Klotho: A novel regulator in female reproductive outcomes and hormone-related cancer. Int J Clin Exp Med 10: 8511-8521, 2017.

37. Xie T, Ye W, Liu J, Zhou L and Song Y: The emerging key role of Klotho in the hypothalamus-pituitary-ovarian axis. Reprod Sci 28: 322-331, 2021

38. Duce JA, Podvin S, Hollander W, Kipling D, Rosene DL and Abraham CR: Gene profile analysis implicates Klotho as an important contributor to aging changes in brain white matter of the rhesus monkey. Glia 56: 106-117, 2008.

39. King GD, Rosene DL and Abraham CR: Promoter methylation and age-related downregulation of Klotho in rhesus monkey. Age 34: 1405-1419, 2012.

40. Semba RD, Moghekar AR, Hu J, Sun K, Turner R, Ferrucci L and O'Brien R: Klotho in the cerebrospinal fluid of adults with and without Alzheimer's disease. Neurosci Lett 558: 37-40, 2014.

41. Rubinek T, Shulman M, Israeli S, Bose S, Avraham A, Zundelevich A, Evron E, Gal-Yam EN, Kaufman B and Wolf I: Epigenetic silencing of the tumor suppressor Klotho in human breast cancer. Breast Cancer Res Treat 133: 649-657, 2012.

42. Wang LJ, Wang X, Wang XJ, Jie P, Lu H, Zhang S, Lin X, Lam EK, Cui Y, Yu J and Jin H: Klotho is silenced through promoter hypermethylation in gastric cancer. Am J Cancer Res 1: 111-119, 2011.

43. Yan Y, Wang Y, Xiong Y, Lin X, Zhou P and Chen Z: Reduced Klotho expression contributes to poor survival rates in human patients with ovarian cancer, and overexpression of Klotho inhibits the progression of ovarian cancer partly via the inhibition of systemic inflammation in nude mice. Mol Med Rep 15: $1777-1785,2017$.

44. Matsumura Y, Aizawa H, Shiraki-Iida T, Nagai R, Kuro-o M and Nabeshima Y: Identification of the human klotho gene and its two transcripts encoding membrane and secreted klotho protein. Biochem Biophys Res Commun 242: 626-630, 1998.

45. Lu L, Katsaros D, Wiley A, de la Longrais IA, Puopolo M and Yu H: Klotho expression in epithelial ovarian cancer and its association with insulin-like growth factors and disease progression. Cancer Invest 26: 185-192, 2008.

46. Wiley A, Katsaros D, Lu L, de La Longrais IAR, Puopolo M, Si J and Yu H: DNA methylation of the human Klotho Gene: Associations with IGF-I, IGF-II, and IGFBP-3 expression and ovarian cancer survival. Cancer Res 66 (Suppl 8): S1204, 2006.

47. Dallol A, Al-Ali W, Al-Shaibani A and Al-Mulla F: Analysis of DNA methylation in FFPE tissues using the MethyLight technology. In: Formalin-Fixed Paraffin-Embedded Tissues: Methods and Protocols. Al-Mulla F (ed). Humana Press, Totowa, NJ, pp191-204, 2011.

48. Dallol A, Buhmeida A, Merdad A, Al-Maghrabi J, Gari MA, Abu-Elmagd MM, Elaimi A, Assidi M, Chaudhary AG, Abuzenadah AM, et al: Frequent methylation of the Klotho gene and overexpression of the FGFR4 receptor in invasive ductal carcinoma of the breast. Tumour Biol 36: 9677-9683, 2015.

49. Losi L, Fonda S, Saponaro S, Chelbi ST, Lancellotti C, Gozzi G, Alberti L, Fabbiani L, Botticelli L and Benhattar J: Distinct DNA methylation profiles in ovarian tumors: Opportunities for novel biomarkers. Int J Mol Sci 19: 1559, 2018.

50. Ebell MH, Culp MB and Radke TJ: A systematic review of symptoms for the diagnosis of ovarian cancer. Am J Prev Med 50: 384-394, 2016.

51. Rooth C: Ovarian cancer: Risk factors, treatment and management. Br J Nurs 22: S23-S30, 2013.

52. Ramaswami R, Bayer R and Galea S: Precision medicine from a public health perspective. In: Annual Review of Public Health. Vol. 39. Fielding JE, Brownson RC and Green LW (eds). Annual Reviews, Palo Alto, CA, pp153-168, 2018.

53. Bachvarov D, L'Esperance S, Popa I, Bachvarova M, Plante M and Têtu B: Gene expression patterns of chemoresistant and chemosensitive serous epithelial ovarian tumors with possible predictive value in response to initial chemotherapy. Int $\mathrm{J}$ Oncol 29: 919-933, 2006 
54. L'Espérance S, Popa I, Bachvarova M, Plante M, Patten N, Wu L, Têtu B and Bachvarov D: Gene expression profiling of paired ovarian tumors obtained prior to and following adjuvant chemotherapy: Molecular signatures of chemoresistant tumors. Int J Oncol 29: 5-24, 2006.

55. Sabatier R, Finetti P, Cervera N, Birnbaum D and Bertucci F: Gene expression profiling and prediction of clinical outcome in ovarian cancer. Crit Rev Oncol Hematol 72: 98-109, 2009.

56. García-Sánchez A and Marqués-García F: Review of methods to study gene expression regulation applied to asthma. In: Molecular Genetics of Asthma. Isidoro García M (ed). Springer New York, NY, pp71-89, 2016.

57. Chan JK, Urban R, Cheung MK, Osann K, Shin JY, Husain A, Teng NN, Kapp DS, Berek JS and Leiserowitz GS: Ovarian cancer in younger vs older women: A population-based analysis. Br J Cancer 95: 1314-1320, 2006.

58. Mohammadian M, Ghafari M, Khosravi B, Salehiniya H, Aryaie M, Bakeshei FA and Mohammadian-Hafshejani A: Variations in the incidence and mortality of ovarian cancer and their relationship with the human development index in European countries in 2012. Biomed Res Ther 4: 1541-1557, 2017.

59. Zheng G, Yu H, Kanerva A, Försti A, Sundquist K and Hemminki K: Familial risks of ovarian cancer by age at diagnosis, proband type and histology. PLoS One 13: e0205000, 2018.

60. Arora N, Talhouk A, McAlpine JN, Law MR and Hanley GE: Long-term mortality among women with epithelial ovarian cancer: A population-based study in British Columbia, Canada. BMC Cancer 18: 1039, 2018.
61. Smith RA, Cokkinides V and Brawley OW: Cancer screening in the United States, 2012: A review of current American Cancer Society guidelines and current issues in cancer screening. CA Cancer J Clin 62: 129-142, 2012.

62. Kim SJ, Rosen B, Fan I, Ivanova A, McLaughlin JR, Risch H, Narod SA and Kotsopoulos J: Epidemiologic factors that predict long-term survival following a diagnosis of epithelial ovarian cancer. Br J Cancer 116: 964-971, 2017.

63. Earp MA and Cunningham JM: DNA methylation changes in epithelial ovarian cancer histotypes. Genomics 106: 311-321, 2015.

64. Köbel M, Bak J, Bertelsen BI, Carpen O, Grove A, Hansen ES, Levin Jakobsen AM, Lidang M, Måsbäck A, Tolf A, et al: Ovarian carcinoma histotype determination is highly reproducible, and is improved through the use of immunohistochemistry. Histopathology 64: 1004-1013, 2014.

This work is licensed under a Creative Commons Attribution-NonCommercial-NoDerivatives 4.0 International (CC BY-NC-ND 4.0) License. 